\title{
Rectosigmoid Region
}

National Cancer Institute

\section{Source}

National Cancer Institute. Rectosigmoid Region. NCI Thesaurus. Code C54188.

The area where the sigmoid colon joins the rectum. 